\title{
Dialogue social et changement institutionnel. Le cas de la mise en œuvre d'une directive européenne
}

\author{
Jean-François Orianne
}

Institut des Sciences Humaines et Sociales (ISHS), Université de Liège, Boulevard du Rectorat 7, B31, B-4000 Liège.

<jforianne@ulg.ac.be>

QRésumé Cet article prend pour objet la mise en œuvre, en Belgique, d'une directive européenne établissant un cadre général relatif à l'information et à la consultation des travailleurs dans la Communauté européenne : la directive 2002/14/CE. L'auteur utilise le «modèle des trois $i$ » en sciences politiques pour analyser la mise en œuvre particulièrement laborieuse qui cristallise un processus de changement ou d'apprentissage institutionnel, perceptible en Belgique, à travers la redéfinition des principales institutions du dialogue social.

Belgique - Dialogue social - Directive européenne - Idée - Institution Interaction-Intérêt.

Gummary Social Dialogue and Institutional Change: The Implementation of a European Directive

The purpose of this article is to analyze the implementation, in Belgium, of the European directive 2002/14/CE establishing a general framework for informing and consulting employees in the European Community. Elaborating on the "model of the three I," the author focuses on the process of change and institutional learning, which has been initiated by this uneasy implementation, through a redefinition of the main institutions of social dialogue in Belgium.

Belgium - European directive - Idea - Institution - Interaction - Interest Social dialogue. 


\section{Introduction}

Cet article prend pour objet la mise en œuvre, en Belgique, d'une directive européenne établissant un cadre général relatif à l'information et à la consultation des travailleurs dans la Communauté européenne : la directive 2002/14/CE.

Les États membres ont bénéficié d'un délai de trois ans pour transposer cette directive en droit national. En Belgique, c'est au lendemain de la date butoir, en mars 2005, que le Conseil national du travail (CNT) remet un avis selon lequel les organisations d'employeurs et de travailleurs n'ont pu parvenir à un accord à ce sujet (cf. Avis $\mathrm{n}^{\circ} 1508$ du CNT). Deux ans plus tard, la Cour européenne de justice de Luxembourg condamne la Belgique pour «manquement d'État». À la suite de cette condamnation en mars 2007, le Gouvernement belge presse instamment les partenaires sociaux de trouver une solution rapide. C'est tout récemment, au terme de sept années d'intense conflit, qu'un accord est enfin conclu, hors de l'arène du $\mathrm{CNT}$, sur une solution complexe et transitoire (cf. Avis $\mathrm{n}^{\circ} 1630$ ).

Ce texte propose d'éclairer ce processus particulièrement laborieux de mise en œuvre en Belgique, dans le cadre duquel se joue la redéfinition substantielle des principales institutions du dialogue social: les conseils d'entreprise, les comités pour la prévention et la protection au travail, et les délégations syndicales.

Cet objet singulier, et relativement circonscrit, offre une occasion originale de tester empiriquement le « modèle des trois $i$ » 1 , autrement dit de prendre en compte les intérêts, les institutions et les idées comme déterminants ou variables de configuration de ce processus de mise en œuvre. L'ambition de ce texte est de souligner l'importance de la dimension processuelle ou procédurale de l'action publique, en introduisant une dimension transversale au modèle analytique : le « $\mathbf{i}$ » de interaction, en référence à la sociologie de George H. Mead. L'apport d'une théorie de l'interaction médiatisée par le langage offre une interprétation plus complète du cas empirique et pose les bases d'une articulation théorique entre les trois « $\mathrm{i}$ ».

Nous commencerons par présenter le «modèle des trois $i$ » en sciences politiques. Nous décrirons ensuite brièvement le cas empirique, avant de procéder à son analyse. Nous conclurons sur l'apport d'une sociologie pragmatique de l'action publique à l'analyse des politiques publiques.

\section{Le « modèle des trois i »}

On assiste, depuis la fin des années 1980, à un renouvellement théorique au sein de l'analyse des politiques publiques, qui s'efforce de dépasser l'opposition classique entre approches "étatiques » (holisme) et approches "pluralistes " (individualisme méthodologique) ${ }^{2}$. Ce renouvellement se caractérise, au sein de la littérature

1. Bruno PALIER et Yves SuREL, «Les "trois i" et l'analyse de l'État en action ", Revue française de science politique, 55 (1), 2005.

2. Peter A HALL, « Policy Paradigms, Social Learning, and the State. The Case of Economic Policymaking in Britain », Comparative Politics, 25 (3), 1993, p. 275-296. 
anglophone, par la redécouverte du concept d'institution ${ }^{3}$ et par un tournant résolument cognitiviste au sein de la littérature francophone ${ }^{4}$. Dans les deux cas, les politiques sont envisagées comme des matrices cognitives et normatives, des cartes mentales, des systèmes de représentation du monde qui façonnent et orientent l'action publique.

Le néo-institutionnalisme renvoie à un ensemble très hétérogène de travaux en sciences politiques. S'efforçant de rompre avec les approches behavioristes et systémiques, ces travaux contribuent directement à redéfinir les institutions comme des processus de mise en ordre réduisant les incertitudes et conflits inhérents à la compétition politique. Peter Hall et Rosemary Taylor ${ }^{5}$ identifient, au sein de ce vaste programme de recherche, trois principaux courants ou trois néo-institutionnalismes: (1) le néo-institutionnalisme historique qui souligne, par exemple, les phénomènes de «dépendance au sentier» (path dependence) ; (2) le néo-institutionnalisme du choix rationnel qui reste attaché à l'héritage de l'école du Public Choice; (3) le néo-institutionnalisme sociologique de Walter Powell et Paul DiMaggio ${ }^{6}$ qui s'écarte le plus explicitement du postulat rationaliste de la sociologie des organisations. Les théoriciens de ce troisième courant néo-institutionnaliste définissent les institutions «de façon beaucoup plus globale que les chercheurs en science politique, de façon à inclure non seulement les règles, procédures ou normes formelles, mais les systèmes de symboles, les schémas cognitifs et les modèles moraux qui fournissent les "cadres de signification" guidant l'action humaine» 7 .

L'approche cognitive des politiques publiques se développe, quant à elle, principalement en France, au départ des travaux anglophones, en particulier ceux de Hugh Heclo (Social Learning), de Peter Hall (Policy Paradigms) et de Paul Sabatier (Advocacy Coalition Framework). Elle s'efforce de rendre compte du poids des idées (au sens large, en ce compris les représentations, les croyances, les valeurs) dans la construction des politiques et, plus largement, dans la conduite de l'action publique. Cette approche cognitive, à mi-chemin entre le fonctionnalisme et le constructivisme ${ }^{8}$, « s'efforce de saisir les politiques publiques comme des matrices cognitives et normatives constituant des systèmes d'interprétation du réel, au sein desquels les différents acteurs publics et privés pourront inscrire leur action ${ }^{9}$. Selon

3. James G. MARCH et Johan P. OLSEN, Rediscovering Institutions : The Organizational Basis of Politics, New York: The Free Press, 1989.

4. Pierre MULLER, «L'analyse cognitive des politiques publiques : vers une sociologie politique de l'action publique ", Revue française de science politique, 50 (2), 2000, p. 189-207.

5. Peter A. HALl et Rosemary C. TAYLOR, « La science politique et les trois néo-institutionnalismes », Revue française de science politique, 47 (3), 1997, p. 469-496.

6. Walter W. Powell et Paul J. DiMaggio (eds.), The New Institutionalism in Organizational Analysis, Chicago : University of Chicago Press, 1991.

7. Ibid., p. 482.

8. Comme le souligne Muller, «si les matrices cognitives sont bien produites par l'interaction d'individusacteurs, elles tendent à s'autonomiser par rapport à leur processus de construction et s'imposer aux acteurs comme modèles dominants d'interprétation du monde " (Pierre MUlLER, "L'analyse cognitive des politiques publiques : vers une sociologie politique de l'action publique », op. cit.).

9. Pierre Muller et Yves Surel, L'analyse des politiques publiques, Paris : Montchrestien, 1998, p. 47. 
Claudio Radaelli ${ }^{10}$, l'intérêt actuel pour la dimension cognitive des politiques publiques est davantage une redécouverte qu'une véritable nouveauté ${ }^{11}$. Selon Paul Sabatier et Edella Schlager 12, cet engouement nouveau aurait moins d'importance aux États-Unis qu'en Europe. En France particulièrement, l'approche cognitive des politiques publiques semble être en vogue depuis quelques années et se développe principalement autour des concepts de référentiel d'action publique 13 et de matrice cognitive et normative 14 .

Ces deux approches, néo-institutionnaliste et cognitive, (re)posent avec insistance la question de l'articulation de trois "variables-clés», "déterminants » ou " dimensions » de l'action publique : les intérêts, les institutions et les idées ${ }^{15}$. Dans un article récent, Bruno Palier et Yves Surel 16 interrogent la pertinence et le caractère opérationnel du modèle heuristique des trois « $\mathrm{i}$ ", tout en soulignant « l'absence d'un véritable système cohérent d'analyse ». Comme le soulignent ces auteurs, l'articulation des trois « $\mathrm{i}$ » est une question théorique qui n'a rien d'original dans la mesure où les intérêts, les idées et les institutions constituent les principales dimensions analytiques « fondatrices » en sciences sociales.

En effet, la célèbre distinction de Vilfredo Pareto entre les actions logiques et les actions non logiques 17 inscrit en sociologie le modèle de l'agir téléologique, qui plonge ses racines dans la philosophie d'Aristote; ce modèle de l'action orientée vers un but se situe au fondement de la théorie des jeux en économie, en sociologie et en psychologie. La sociologie d'Émile Durkheim se définit, quant à elle, explicitement comme la science des institutions sociales 18 : l'institution familiale, scolaire, totémique, etc. Enfin, la sociologie de Max Weber, en particulier sa Sociologie des religions, pose la question du poids des idées religieuses ou des conceptions du monde sur le comportement économique de diverses sociétés. Dans L'éthique protestante et l'esprit du capitalisme, Weber précise la nature de son projet sociologi-

10. Claudio RADAELLI, «Logiques de pouvoirs et récits dans les politiques publiques de l'Union européenne ", Revue française de science politique, 50 (2), 2000.

11. Radaelli rappelle à juste titre les travaux «pionniers » de Lindblom (l'incrémentalisme), de Simon (la rationalité limitée), d'Axelrod (les cartes cognitives des élites politiques) et de Deutsch (la politique comme apprentissage).

12. Paul A. SABAtier et Edella SCHLAGER, «Les approches cognitives des politiques publiques : perspectives américaines ", Revue française de science politique, 50 (2), 2000, p. 209-234.

13. Bruno JoвERT, « Représentations sociales, controverses et débats dans la conduite des politiques publiques ", Revue française de science politique, 42 (2), 1992, p. 219-234.

14. Pierre MulLer et Yves Surel, L'analyse des politiques publiques, op. cit.

15. Hugh Heclo, «Ideas, Interests, and Institutions", in Lawrence C. DodD et Calvin JiLlson (eds.), The Dynamics of American Politics. Approaches and Interpretations, Boulder: Westview Press, 1994 ; Peter A. HALL, " The Role of Interests, Institutions and Ideas in the Comparative Political Economy of the Industrialized Nations ", in Mark I. LichbaK et Alan S. Zuckerman (eds.), Comparative Politics, Rationality, Culture and Structure, Cambridge: Cambridge University Press, 1997, p. 174-207; Yves SuREL, "Idées, intérêts, institutions dans l'analyse des politiques publiques ", Pouvoirs, 87, 1998, p. 161-178.

16. Bruno PALIER et Yves SUREL, «Les “trois I” et l'analyse de l’État en action », art. cité., p 7-32.

17. Cf. Vilfredo PARETO, Traité de sociologie générale [1917], Paris, Genève : Librairie Droz, 1968, p. 67-68.

18. Cf. Émile Durkheim, Les règles de la méthode sociologique, Paris: PUF, 1950, préface de la seconde édition, p. XIV-XV. 
que : " contribuer [...] à faire comprendre de quelle façon les "idées" deviennent des forces historiques efficaces $» 19$.

Si la question n'a rien d'original, c'est bien parce qu'elle est essentielle. Elle s'enracine en effet dans la première grande synthèse théorique en sociologie : la sociologie de l'action de Talcott Parsons ${ }^{20}$. Parsons se plaît à rappeler, dans plusieurs écrits, son triple héritage de Pareto, Durkheim, Weber, et l'intuition forte d'une convergence, entre ces auteurs, qui lui permet d'appréhender la « structure de l'action sociale ». Parsons définit l'action comme un comportement intentionnel, orienté vers des buts, des fins, des anticipations ; elle est réglée par des normes ; elle est située ; elle implique une dépense d'énergie, une motivation 21.

Sans grande originalité, le «modèle des trois $\mathbf{i}$ » en sciences politiques repose donc cette question cruciale de l'articulation de trois dimensions-clés de l'action humaine identifiées par trois «fondateurs" de la sociologie. Ce qui est plus singulier, c'est l'omission d'un quatrième fondateur: G.H. Mead qui tiendrait à lui seul un quatrième « $\mathrm{i}$ » dans le modèle analytique, celui de l'interaction médiatisée par le langage et orientée vers l'intercompréhension. Cette quatrième contribution majeure à la théorie sociologique de l'action humaine n'a d'ailleurs pas échappé à Parsons 22.

Le modèle des trois $i$, en sciences politiques, semble négliger l'interaction humaine ${ }^{23}$, et l'intercompréhension n'est pas thématisée comme une dimension à part entière de l'action (publique), irréductible aux intérêts, aux institutions ou aux idées (dans leur dimension substantielle) 24. Or, comme Habermas l'a bien montré dans sa théorie de l'agir communicationnel, la sociologie de G.H. Mead pose les bases d'une théorie à part entière de l'action : l'interaction d'au moins deux sujets,

19. Max WeBER, L'éthique protestante et l'esprit du capitalisme, Paris : Plon,1964, p. 102.

20. Talcott PARSONS, The Structure of Social Action, New York: Mac Graw-Hill, 1937; Talcott PARSONS et Edward SHILS (eds.), Toward a General Theory of Action, Cambridge (Mass.) : Harvard University Press, 1957.

21. François Bourricaud, L'individualisme institutionnel, Paris : PUF, 1977.

22. En effet, dans sa formulation des quatre sous-systèmes de l'action humaine, Parsons réserve une case à chacun de ses grands maîtres : la case A (adaptation) pour le behaviorisme social de Mead (le comportement comme organisme et le langage comme medium); la case G (goal attainment) pour l'agir téléologique de Pareto (les buts, les intérêts individuels et les stratégies collectives) ; la case I (intégration) pour la sociologie durkheimienne (le poids des normes et leur fonction d'intégration) ; la case L (lattent pattern maintenance) pour la sociologie wébérienne (le poids des valeurs et la fonction de régulation du système culturel). Joas (Hans JOAS, La créativité de l'agir, Paris : éd. du Cerf, 1999) insiste cependant sur le traitement problématique que Parsons réserve à la sociologie de Mead, en particulier la difficulté qu'il éprouve à intégrer le pragmatisme américain (et sa critique en profondeur du modèle rationnel) dans sa théorie de l'action.

23. Sur ce point, Christine Musselin souligne l'écart épistémologique entre, d'une part, l'analyse des politiques publiques centrée principalement sur la décision ou le discours des élites et, d'autre part, la sociologie de l'action publique centrée sur l'interaction d'une multitude d'acteurs : cf. Christine MuSSELIN, "Sociologie de l'action organisée et analyse des politiques publiques: deux approches pour un même objet ? ", Revue française de science politique, 55 (1), 2005, p. 51-71.

24. À l'exception de quelques travaux particulièrement novateurs, comme par exemple ceux de Yves Sintomer et Jacques DE MAILlARD, «The limits to local participation and deliberation in the French "politique de la ville" ", European Journal of Political Research, 46 (4), 2007, p. 503-529 ; de Philippe URFALINO, «La délibération n'est pas une conversation. Délibération, décision collective et négociation ", Négociations, 2, 2005, p. 99-114; ou encore de Jon ELSTER, «L'usage stratégique de l'argumentation », Négociations, 2, 2005, p. $59-82$. 
capables de parler et d'agir, qui engagent une relation interpersonnelle. Dans le modèle de l'agir communicationnel, « les acteurs recherchent une entente sur une situation d'action, afin de coordonner consensuellement leurs plans d'action et de là même leurs actions » 25 . La notion centrale d'interprétation met au premier plan l'idée d'une négociation sur la définition des situations susceptibles de consensus. En clair, dans ce modèle, l'action n'est pas a priori orientée vers une finalité, ni régulée par des normes, ni motivée ou façonnée par des idées ou des valeurs ; l'(inter-)action est ici orientée vers l'intercompréhension, vers la production collective d'idées ou de valeurs, de normes et de finalités. Le langage, tout à fait central dans ce modèle, apparaît comme le medium le plus général à travers lequel circulent les valeurs, les normes et les intérêts.

La prise en compte de cette quatrième «variable " (l'interaction médiatisée par le langage) présente un double intérêt, empirique et théorique, en tant que lieu d'articulation entre les trois i, à savoir la dimension processuelle (selon Mead) ou procédurale (selon Habermas) de l'action publique. Mais avant de procéder à l'analyse du cas empirique, il convient d'en présenter brièvement ses traits singuliers.

\section{Présentation du « cas » empirique : la directive européenne 2002/14/CE et sa non-transposition en droit belge}

Le 11 mars 2002, le Parlement européen et le Conseil de l'Union européenne ont arrêté la directive 2002/14/CE établissant un cadre général relatif à l'information et à la consultation des travailleurs dans la Communauté européenne. Cette directive s'inscrit dans le sillage de deux autres directives visant également à promouvoir le dialogue social en Europe: les directives 94/45/CE du Conseil du 22 septembre 1994 (comité d'entreprise européen) et 2001/86/CE du Conseil du 8 octobre 2001 (implication des travailleurs).

La « cadette des trois sœurs » - pour reprendre l'expression de Roger Blanpain fixe des exigences minimales en matière d'information et de consultation des travailleurs. L'information y est définie comme «la transmission par l'employeur de données aux représentants des travailleurs "; la consultation, quant à elle, implique "l'échange de vues et l'établissement d'un dialogue entre les représentants des travailleurs et l'employeur ».

L'article 3 définit le champ d'application de la directive : elle s'applique, selon le choix fait par les États membres, soit aux entreprises de 50 travailleurs et plus, soit aux établissements employant au moins 20 travailleurs. La notion d'entreprise inclut toute entreprise publique ou privée exerçant une activité économique et poursuivant ou non un but lucratif. Le terme établissement est défini comme l'« unité d'exploitation ». Cette distinction terminologique est cruciale et lourde de conséquences sur un plan juridique puisqu'elle affecte directement le seuil d'occupation nécessaire pour l'application de la directive ${ }^{26}$. C'est sur ce point, purement formel,

25. Jürgen HABERMAS, Théorie de l'agir communicationnel, tome 1 : Rationalité de l'agir et rationalisation de la société, traduit de l'allemand par Jean-Marc Ferry, Paris : Fayard, 1987, p. 102.

26. Filip DoRSSEMONT, « Kaderrichtlijn Informatie en Raadpleging : een hefboom voor meer werknemersinspraak in Belgische KMO's ? ", Doctrine, 116, 15 juin 2005, p. 758-764. 
que se cristallise, en Belgique, un vif débat au sein des partenaires sociaux : faut-il ou non abaisser le seuil national (de 50 à 20) ? Faut-il ou non se servir de la directive pour introduire des organes paritaires dans les "petites" entreprises à partir de 20 travailleurs?

En règle générale, la transposition d'une directive européenne (relevant de la «DG emploi et affaires sociales ") en droit belge s'opère grosso modo de la manière suivante: (1) le ministre fédéral de l'Emploi sollicite l'avis du Conseil national du travail $(\mathrm{CNT}) 27$, conformément aux recommandations du Conseil de l'Europe (l'avis des partenaires sociaux comme procédure de mise en œuvre) ; (2) le CNT émet un avis : a) il sélectionne les éléments juridiques de la directive qui doivent faire l'objet d'une transposition en droit belge, et justifie, pour le reste, de l'existence d'un cadre légal conforme à la directive ; b) il départage les matières faisant l'objet d'une transposition conventionnelle des matières faisant l'objet d'une transposition légale ; (3) si l'avis est unanimement favorable, le CNT élabore une convention collective de travail ; parallèlement, s'il y a lieu, le législateur (au sens large du terme) édicte une loi. Quand le CNT ne parvient pas à trouver un accord consensuel - comme dans le cas qui nous occupe -, le processus de mise en œuvre est paralysé.

Les États membres ont bénéficié d'un délai d'un peu plus de trois ans (jusqu'au 23 mars 2005) pour traduire en droit national ces exigences minimales prises au niveau européen (article 11). En Belgique, le CNT a remis le 24 mars 2005 un avis selon lequel les organisations d'employeurs et de travailleurs n'avaient pu parvenir à un accord à ce sujet. Sur les plus de 1500 avis rendus par le CNT depuis sa création en 1952, il s'agit plutôt d'un cas exceptionnel, comme nous le confient en entretien les représentants qui y siègent 28 .

L'absence de transposition de la directive a conduit le syndicat chrétien (CSC) à introduire, le 20 avril 2006, une plainte formelle devant la Commission européenne; deux mois plus tard, la Commission, à son tour, citait la Belgique devant la Cour européenne de justice. La Cour européenne de justice de Luxembourg est officiellement saisie du dossier le 20 juillet. Le 29 mars 2007, elle rend son arrêt et condamne la Belgique (ainsi que le Grand-duché de Luxembourg) pour «manquement d'État », pour ne pas avoir transposé la directive 2002/14.

Il convient dès à présent d'utiliser ce cas empirique singulier pour tester le modèle des trois $i$, autrement dit articuler les trois dimensions analytiques de l'action publique pour tenter de comprendre ce blocage institutionnel ainsi que son issue provisoire au terme de sept années de conflit entre les partenaires sociaux. Le ma-

27. Le Conseil national du travail (CNT) et le Conseil central de l'économie (CCE) constituent en Belgique les deux institutions « coupole» du dialogue social. C'est la loi organique du 29/05/1952 qui institue la CNT comme établissement public dont la mission principale consiste à adresser des avis ou propositions à un ministre ou aux chambres législatives. En outre, depuis la loi du 5 décembre 1968 sur les conventions collectives de travail et les commissions paritaires, le CNT a également le pouvoir de conclure des conventions collectives de travail, soit pour l'ensemble des secteurs d'activités économiques, soit pour l'un de ces secteurs. Le CNT se compose de représentants, en nombre égal, des principales organisations des employeurs et des travailleurs.

28. Les agents interrogés évoquent également l'avis relatif au congé éducatif et celui sur le harcèlement au travail comme autres cas de figure exceptionnels de dissension au sein du CNT. 
tériau empirique se compose d'une dizaine d'entretiens des principaux représentants des travailleurs et des employeurs siégeant au CNT et en charge du dossier ; ils ont été, pour la plupart, interrogés à deux reprises, en 2006 (sur leur désaccord : Avis $n^{\circ} 1508$ ) et en 2008 (sur leur accord : Avis $n^{\circ} 1630$ ). En outre, une analyse documentaire ciblée a permis de reconstruire la trame argumentative et les principales coordonnées officielles du débat et leur évolution.

\section{III. « i » comme intérêts}

L'institutionnalisme du choix rationnel - en particulier les travaux pionniers portant sur l'étude des comportements au sein du Congrès des États-Unis - fournit un cadre d'analyse pertinent pour décrire l'institution que représente le Conseil national du travail en Belgique, cette institution qui naît d'une loi organique en 1952 à la suite de plusieurs organismes dont les plus anciens remontent à la fin du $\mathrm{XIX}^{\mathrm{e}}$ siècle 29 . On pourrait partir de ce même "paradoxe significatif» qui amena les chercheurs américains (comme Riker, Mc Celvey, Fiorina, etc.) à redécouvrir le concept d'institution. Comment, en effet, expliquer une majorité d'avis consensuels au sein du CNT compte tenu des intérêts divergents des membres qui y siègent, d'une tradition conflictualiste de la négociation collective au sein des partenaires sociaux et du caractère multidimensionnel des questions à traiter ? Selon le modèle théorique de l'institutionnalisme du choix rationnel, le CNT en tant qu'institution peut être expliqué par le fait qu'il abaisse les coûts de transactions liés à la conclusion d'accords (les avis) et de conventions (les conventions collectives de travail, CCT) de façon à permettre aux multiples représentants de travailleurs et d'employeurs de tirer des bénéfices de l'échange, ce qui rend possible l'adoption d'avis de CCT relativement stables 30 .

Selon cette perspective analytique, le droit ou plus globalement les règles ou les institutions sont envisagés comme des ressources (au service de stratégies d'acteurs ${ }^{31}$ ) : des ressources de coordination, des outils, des instruments pour « contrôler des zones d'incertitude» (selon l'heureuse expression de Michel Crozier) dans un champ de rapports de pouvoir. Dans le cas de la transposition de la directive européenne 2002/14/CE en Belgique, la principale zone d'incertitude concerne la délimitation d'un seuil minimal pour la création d'organes paritaires au sein de l'entreprise : le choix laissé par la Commission de fixer le seuil à 20 (au niveau de l'établissement) ou à 50 (au niveau de l'entreprise). Cette incertitude liée à la liberté de choix laissée aux États membres est renforcée par la relative imprécision des

29. La Commission du travail (créée en 1886), les Conseils de l'industrie et du travail (1887), le Conseil supérieur du travail (1892) et le Conseil supérieur du travail et de la prévoyance sociale (1935). Après la Seconde Guerre mondiale, une place importante est réservée aux représentants du monde économique et social : ce mouvement participatif s'illustre par l'institution, en 1948, du Conseil central de l'économie et du Conseil national du travail en 1952 (succédant au Conseil paritaire général mis en place depuis 1944).

30. Paraphrasant Peter A. HALL et Rosemary C. TAYLOR, «La science politique et les trois néoinstitutionnalismes", op. cit., p. 477.

31. Cf. Jacques COMMAILLE, «La négociation comme expression d'une nouvelle économie de la légalité », in Didier VRancken, Christophe Dubois et Frédéric Schoenaers (dir.), Penser la négociation, Bruxelles: De Boeck, 2008, p. 39-49. 
concepts utilisés par la Commission et, dès lors, la marge d'interprétation laissée aux acteurs. Dès lors, le droit positif belge et l'institution du CNT constituent des ressources-clés pour réduire l'incertitude en contrôlant les marges d'autonomie laissée (ou formée) par la norme européenne.

Dans le cas qui nous occupe, le but du «jeu» au sein du CNT peut être résumé de la manière suivante : il s'agit, pour les représentants des travailleurs, d'avancer des " pions » dans l'entreprise ; et à l'inverse, pour les représentants du banc patronal, de "repousser l'attaque» ou du moins cette "tentative d'invasion ». L'Avis $\mathrm{n}^{\circ} 1508 \mathrm{du}$ CNT, rendu le 24/03/2005, acte les positions divergentes des membres du Conseil sur la question de la transposition de la directive 2002/14/CE en Belgique :

a) la position commune des syndicats socialistes (FGTB) et socio-chrétiens (CSC) défend la solution de l'abaissement du seuil national (à 20 travailleurs) pour la création d'organes paritaires au sein de l'entreprise ;

b) la position commune du «banc patronal» (UCM-UNIZO) s'oppose fermement à cette option et défend celle d'un seuil national fixé à 50 pour la création d'organes paritaires au sein de l'entreprise ;

c) la position du syndicat libéral (CGSLB) défend une solution intermédiaire : ne pas abaisser le seuil national, mais étendre les compétences de la délégation syndicale (dans les entreprises qui comptent au moins 20 travailleurs) et modifier leur composition.

La position commune de la CSC et de la FGTB peut être lue aisément comme une position opportuniste ou intéressée : les acteurs se servent de la directive européenne pour abaisser le seuil national et, corrélativement, se servent du droit national pour contrôler les marges d'incertitude laissées par la directive. Cette lecture stratégique correspond grosso modo à l'interprétation spontanée que les acteurs du CNT livrent en entretien. Comme l'explique ce représentant du banc patronal (UCM), « les syndicats cherchent par tous les moyens à utiliser la directive pour forcer la barrière syndicale au niveau des petites entreprises et abaisser les seuils pour la création d'organes paritaires (CE et CPPT)».

Selon le syndicat chrétien, la non-transposition de la directive priverait plus d'un million et demi de travailleurs belges du droit à être consultés et informés. On peut estimer aisément l'effet quantitatif que produirait la mise en œuvre en Belgique de la directive 2002/14/CE : si le seuil de 50 travailleurs est retenu, l'information et la consultation des travailleurs couvriraient, en théorie, $60 \%$ de l'emploi salarié (dans 4,65\% des entreprises) ; si le seuil de 20 travailleurs est retenu, la couverture s'élèverait à $75,7 \%$ de l'emploi salarié (dans $11,8 \%$ des entreprises) ${ }^{32}$. Les effets potentiels de la mise en œuvre ne sont donc pas négligeables.

La position commune de la CSC et de la FGTB n'est pas la seule à être lue spontanément (par les acteurs) comme une position intéressée. En effet, la position du syndicat libéral (CGSLB) comporte également une dimension stratégique - comme leurs adversaires ne manquent pas de le souligner - cherchant avant tout à se posi-

32. Source : ONSS, situation au 21 décembre 2005. 
tionner dans un champ de rapports de force où ils sont largement minoritaires ${ }^{33}$. Comme l'explique ce représentant de la CSC : "Au début, c'était difficile de négocier avec la CGSLB .[...] La CGSLB poussait pour être désignée partout, dans tous les secteurs, alors que dans certains secteurs, comme le gardiennage ou le textile, ils ne sont pas représentatifs et, dès lors, ne peuvent pas créer de délégation. "

La position de banc patronal n'échappe pas non plus à cette lecture stratégique : les représentants des travailleurs ont longuement insisté, en entretien, sur l'intérêt de UCM-UNIZO de bloquer la décision au sein du CNT. Pour la CSC, il semble clair que «l'UNIZO et l'UCM avaient intérêt à ce qu'il n'y ait pas d'accord conclu au sein du CNT. Ils évitaient clairement la discussion, car ils ont eu l'assurance de la part du Gouvernement qu'il n'y aurait pas de décision unilatérale de changement de seuil en l'absence d'un accord au sein du CNT. Donc, pour eux, chaque retard était considéré comme un gain de temps".

Bien qu'elle se fonde sur une conception un peu caricaturale de l'acteur, cette lecture stratégique «spontanée » du conflit est essentielle dans la mesure où elle fait prendre conscience, aux acteurs publics comme aux chercheurs, que le débat au sein du CNT n'est pas un débat purement juridique ou technique. «La question du seuil, on peut la voir à deux niveaux. Il y a l'aspect strictement juridique, technique : c'est un doute sur l'interprétation de la directive. Quel seuil retenir ? Et puis, il y a l'aspect politique, le plus important, selon nous » (FGTB). Cependant, la dimension "politique » du débat peut-elle être réduite à sa dimension "stratégique » ou instrumentale ? Et quand bien même il en serait ainsi, encore faudrait-il être en mesure d'expliquer comment ces intérêts surgissent.

Les théories utilitaristes restent souvent silencieuses sur l'origine des intérêts des acteurs ${ }^{34}$ : d'où nous viennent-ils, pourquoi ou comment a-t-on des intérêts ou des idées auxquelles on accorde de la valeur? La sociologie de G.H. Mead apporte des éléments de réponse convaincants, en interrogeant le processus par lequel ces intérêts ou ces idées se forment: ce processus est celui de l'interaction humaine. On peut dès lors relire les prises de position «intéressées » des différents acteurs en présence au sein du CNT comme autant de gestes - qui manifestent des attitudes (attaque, défense, repli stratégique, feinte, etc.) - par lesquels les acteurs font naître, sollicitent en eux-mêmes les réponses qu'ils suscitent chez les autres. Selon Mead, ce processus, qui conduit tendanciellement les acteurs à agir les uns comme les autres, repose sur la réflexivité de l'« animal humain» - condition essentielle du développement de l'esprit. C'est ainsi que les positions respectives des acteurs syndicaux adoptent (anticipent et synthétisent) toutes le point de vue de l'autre: elles sont le produit de leur environnement, d'un univers de discours particulier. Le coup de A (CSC-FGTB) anticipe la réponse de B ; le coup de B (UCM-UNIZO) adop-

33. Depuis les dernières élections sociales en 2004, la CGSLB occupe 5,84 \% des sièges CPPT et $6,09 \%$ des sièges CE.

34. Contrairement à la sociologie de Michel Crozier et de ses collègues (Ehrard Friedberg, Jean-Daniel Reynaud, Olgierd Kuty, etc.) qui s'est toujours attachée à rendre compte des systèmes d'action concrets, des règles du jeu d'un ordre local, pour expliquer comment émergent les intérêts des acteurs. 
te le point de vue de A; le coup de C (CGSLB) constitue une synthèse partielle (et partiale) des coups respectifs de A et B.

Cependant, le processus interactif qui se joue au sein du CNT n'est pas un simple «jeu libre» (le play) où les différentes réponses ne s'aligneraient que sur celles des autres. Il s'agit d'un « jeu réglementé » (le game) où l'alignement s'opère également sur un autrui généralisé. Pour le dire autrement, l'interaction n'est pas seulement médiatisée par des symboles significatifs ; elle est aussi régulée par des normes, par des institutions : d'une part, les institutions européennes à l'origine de la directive et, d'autre part, les institutions du dialogue social en Belgique. Signalons cependant que ce jeu réglementé relève davantage du modèle du "jeu glissant" que de celui du " jeu fixe» 35 dans la mesure où ce qui se joue au sein du CNT n'est autre que la transformation des règles du jeu elles-mêmes 36 ; le terrain est glissant lui aussi, car ce jeu ponctuel sur les règles s'inscrit dans un contexte plus large de transformation des règles du système de relations professionnelles en Belgique, jusqu'alors caractérisé par une très grande autonomie des partenaires sociaux 37 .

\section{IV. « $i$ » comme institutions}

La deuxième dimension analytique du «modèle des trois $\mathrm{i}$ » nous renvoie à la tradition durkheimienne, en sociologie, selon laquelle le droit, les règles, les institutions sont envisagés comme des contraintes (assorties de sanctions) qui pèsent sur l'action. Ces règles peuvent être formelles ou informelles, légales ou conventionnelles, écrites ou non écrites. Quant aux sanctions, elles peuvent être explicites ou implicites, punitives ou restitutives, etc.

Nous commencerons par évoquer brièvement la contrainte exercée par les institutions européennes sur l'État belge. Nous présenterons ensuite, de manière très succincte, le dispositif belge de concertation sociale institué au niveau de l'entreprise, en tant que cadre formel relativement contraignant dans lequel doit s'insérer la directive. Ces deux premiers éléments renvoient à la dimension substantielle des institutions qui contraignent et habilitent les acteurs du dialogue social en Belgique. Un dernier élément, d'ordre procédural ou processuel, complètera l'analyse : il ne s'agit pas ici d'une contrainte substantielle, mais bien d'une routine, un phénomène complexe de « dépendance au sentier » 38 qui pèse lourdement sur la décision actuelle de transposer cette directive en droit belge.

35. Gérard ADAM et Jean-Daniel REYNAUD, Conflits du travail et changement social, Paris : PUF, 1978.

36. Jean-Daniel ReYNAud, Les règles du jeu. L'action collective et la régulation sociale, Paris : Armand Colin, 1989.

37. Olgierd KuTY, "La régulation belge. Hypothèses pour une première conceptualisation», Working paper du CRIS, Institut des Sciences Humaines et Sociales, Université de Liège, 2006. Comme le montre bien O. Kuty, la politique européenne en matière d'emploi a désormais renforcé le rôle de l'acteur gouvernemental en instaurant une surveillance multilatérale - notamment par le mécanisme des Plans d'actions nationaux - responsabilisant ainsi davantage les États membres que les partenaires sociaux.

38. Bruno PAliER et Giuliano Bonoli, «Phénomènes de path dependence et réformes des systèmes de protection sociale", Revue française de science politique, 49 (3), 1999, p. 399-420 ; Douglass C. NoRTH, Institutions, Institutional Change, and Economic Performance, Cambridge: Cambridge University Press, 1990. 


\section{IV.1. La contrainte européenne}

Si les règlements européens ont une portée générale, s'ils sont obligatoires et directement applicables dans tout État membre, en revanche les directives européennes - prises par le Parlement conjointement avec le Conseil, par le Conseil ou par la Commission - ne lient les États membres destinataires que dans le cadre des objectifs qu'elles poursuivent, tout en laissant aux instances nationales la compétence quant à la forme et aux moyens (article 249 du Traité). En clair, les institutions européennes laissent aux États membres le soin de choisir les mesures nationales de transposition appropriées permettant la concrétisation de ces objectifs.

En général, un délai est fixé pour la transposition de la directive dans la législation nationale des différents États membres. Comme le souligne Bernd Shulte, " dans certaines circonstances, l'absence de transposition d'une directive par le législateur national peut donner lieu à une requête de réparation basée sur le droit communautaire, que peut faire valoir un bénéficiaire de la directive préjudicié du fait de l'omission de la transposition au détriment de l'État membre négligent » 39 .

En Belgique, nous l'avons vu, l'absence de transposition de la directive 2002/14/CE a donné lieu à une telle requête de réparation. Le 20 avril 2006, la CSC introduit une plainte formelle devant la Commission européenne et, deux mois plus tard, la Commission cite la Belgique devant la Cour européenne de justice (affaire C-320/06). La Cour européenne de justice de Luxembourg, officiellement saisie du dossier le 20 juillet, rend son arrêt le 29 mars 2007 et condamne la Belgique pour "manquement d'État». La sanction se limite à un simple «rappel à l'ordre ", sans conséquence immédiate sur un plan financier. Cependant, une procédure de mise en demeure peut être lancée par la Commission (sur base de l'article $228 \mathrm{du}$ Traité européen) si la Belgique ne transpose pas la directive dans les meilleurs délais: c'est donc la menace d'une amende de 3 millions d'euros (et d'astreintes pouvant s'élever jusqu'à 210.000 euros par jour de retard) qui pèse sur l'État belge.

La norme européenne ne constitue pas la seule contrainte qui pèse sur la décision. Il faut aussi rendre compte du cadre contraignant et habilitant du droit positif belge, dans lequel la directive doit être transposée.

\section{IV.2. Les institutions du dialogue social en Belgique}

Comment expliquer un tel blocage institutionnel - relatif à la transposition d'une directive qui se borne à fixer des exigences minimales en matière d'information et de consultation des travailleurs - dans un contexte national, célèbre pour son " art du compromis", où la concertation sociale est instituée depuis plus de 60 ans?

En effet, les premiers organes paritaires de participation des travailleurs au sein de l'entreprise ont été institués en Belgique, au lendemain de la Seconde Guerre mondiale. La section IV de la loi du 20/09/1948 portant sur l'organisation de l'économie institue des conseils d'entreprise (CE) « dans toutes les entreprises occupant

39. Bernd Schulte, « Droit social européen », Revue belge de sécurité sociale, 4 e trimestre 2001, p. 667. 
habituellement en moyenne au moins 50 travailleurs" (article 14). Le législateur précise qu'il y a lieu d'entendre par entreprise : "l'unité technique d'exploitation, définie $[. .$.$] à partir des critères économiques et sociaux ; en cas de doute, ces der-$ niers prévalent. Le Roi prescrit une procédure à suivre pour déterminer paritairement la notion d'unité technique d'exploitation [...]» (op. cit.) ${ }^{40}$.

Les conseils d'entreprise ne constituent pas les seuls organes paritaires de concertation en Belgique institués au niveau de l'entreprise. Depuis 1996, des comités pour la prévention et la protection au travail (CPPT) ont également été institués par le législateur, dans le cadre de la loi du 04/08/1996 relative au bien-être des travailleurs lors de l'exécution de leur travail. Le chapitre VIII de cette loi traite spécifiquement des CPPT : «Des comités sont institués dans toutes les entreprises occupant habituellement en moyenne au moins 50 travailleurs. Dans les mines, minières et carrières souterraines, ces comités sont institués dès que celles-ci occupent habituellement 20 travailleurs en moyenne" (article 49). Comme dans la loi de 1948, le législateur entend l'entreprise comme "l'unité technique d'exploitation $» 41$.

Un point important est à noter : lorsqu'il n'existe pas de comité dans l'entreprise, la délégation syndicale est chargée d'exercer les missions qui lui sont attribuées (article 52) ; dans les entreprises où il n'existe ni un comité ni une délégation syndicale, cette tâche revient alors directement aux travailleurs eux-mêmes (article 53). Rappelons que les délégations syndicales, contrairement aux CE et CPPT, n'ont jamais été instituées par le législateur. C'est la CCT $n^{\circ} 5$, conclue pour une durée indéterminée au sein du CNT, qui règle leur statut ${ }^{42}$. Elle est une des rares CCT à n'avoir jamais été rendue obligatoire par arrêté royal. Ces normes conventionnelles n'ont donc pas force de loi : en clair, il n'existe pas en Belgique de systè-

40. L'article 15 de cette même loi définit les missions des conseils d'entreprise : a) donner leur avis et formuler toutes suggestions ou objections sur toutes mesures qui pourraient modifier l'organisation du travail, les conditions de travail et le rendement de l'entreprise ; b) recevoir du chef d'entreprise des informations économiques et financières ; $c$ ) élaborer et modifier le règlement d'atelier ou le règlement d'ordre intérieur de l'entreprise ; d) veiller à la stricte application de la législation industrielle et sociale protectrice des travailleurs ; veiller à l'application de toute disposition générale intéressant l'entreprise ; e) examiner les critères généraux à suivre, en cas de licenciement et d'embauchage des travailleurs; f) fixer les dates de vacances annuelles et établir, s'il y a lieu, un roulement du personnel.

Les élections pour les conseils d'entreprise ont lieu tous les quatre ans (article 21). Retenons également que le conseil d'entreprise se réunit au siège de l'entreprise ; il est présidé par le chef d'entreprise ou par son délégué à la présidence ; il est convoqué au moins une fois par mois à la diligence du chef d'entreprise ou (d'un tiers) des membres du conseil représentant le personnel (article 22).

41. Le CPPT a essentiellement pour mission «de rechercher et de proposer tous les moyens et de contribuer activement à tout ce qui est entrepris pour favoriser le bien-être des travailleurs lors de l'exécution de leur travail" (article 65). Le législateur entend par "bien-être" «l'ensemble des facteurs concernant les conditions dans lesquelles le travail est effectué» (article 3 ). Il a trait principalement à : «1) la sécurité du travail ; 2) la protection de la santé du travailleur au travail ; 3) la charge psycho-sociale occasionnée par le travail ; 4) l'ergonomie ; 5) l'hygiène du travail ; 6) l'embellissement des lieux de travail ; 7) les mesures prises par l'entreprise en matière d'environnement ; 8) la protection des travailleurs contre la violence et le harcèlement moral ou sexuel au travail » (article 4). Les élections pour les comités ont également lieu tous les quatre ans (article 58).

42. La CCT $n^{\circ} 5$ du 24/05/1971 concernant le statut des délégations syndicales du personnel des entreprises (modifiée et complétée par la CCT $n^{\circ} 5$ bis du 30/06/1971 et $n^{\circ} 5$ ter du 21/12/1978) définit les principes essentiels concernant la compétence et les modalités de fonctionnement des délégations syndicales. 
me effectif de protection des délégués syndicaux en entreprise ni de procédure effective de sanction en cas de licenciement d'un délégué pour un motif lié à l'exercice de son mandat.

En résumé, si le législateur n'a jamais reconnu officiellement l'existence des délégations syndicales, en revanche il a doté les entreprises (à partir de 50 travailleurs) de véritables structures de concertation sociale permanente portant sur les activités économiques de l'entreprise et le bien-être des travailleurs : d'une part, les conseils d'entreprise (CE) et, d'autre part, les comités pour la prévention et la protection au travail (CPPT).

Les institutions du dialogue social en Belgique

\begin{tabular}{|c|c|c|}
\hline Niveau & Type d'organe paritaire & Cadre légal \\
\hline $\begin{array}{c}\text { National et } \\
\text { interprofessionnel }\end{array}$ & $\begin{array}{c}\text { Conseil national du travail (CNT) } \\
\text { Conseil central de l'économie (CCE) }\end{array}$ & Loi du 29/05/1952 \\
\hline Sectoriel & Commissions paritaires (CP) & Loi du 05/12/1968 \\
\hline Entreprise & $\begin{array}{c}\text { Conseil d'entreprise (CE) } \\
\text { Conseil pour la prévention et la protection } \\
\text { au travail (CPPT) }\end{array}$ & Lo/09/1948 \\
\hline
\end{tabular}

\section{IV.3. Le processus de dépendance au sentier}

À la lecture de cette description sommaire du dispositif belge de concertation sociale (cf. schéma ci-dessus), on a du mal à comprendre où se situe le problème : d'abord, la Belgique semble répondre plus que parfaitement aux exigences minimales fixées par les institutions européennes; ensuite, le seuil national, fixé à 50 travailleurs au niveau de l'entreprise, laisse peu de doute sur l'interprétation qui doit être faite des modalités de choix laissées par la Commission. La question de l'interprétation sera traitée dans le point suivant. Commençons déjà par éclaircir le premier point.

Si la loi de 1948 fixe officiellement à 50 travailleurs le seuil pour la création d'un conseil d'entreprise (l'organe dont les missions s'apparentent le plus à celles fixées par les institutions européennes), en pratique ce seuil n'a jamais été atteint en Belgique. À y regarder de plus près, on s'aperçoit que l'article 28 de cette fameuse loi de 1948 permet au Roi de retarder l'entrée en vigueur des dispositions sur l'institution d'un conseil d'entreprise dans les entreprises occupant moins de 200 travailleurs ${ }^{43}$. Notons que, 60 ans plus tard, le seuil légal de 50 travailleurs n'est toujours pas atteint. Lors de chaque élection sociale (soit tous les quatre ans depuis 1948), un arrêté royal fixant la procédure électorale détermine le seuil à respecter. Les arrêtés d'application de la loi ont progressivement fait descendre le seuil pour s'arrêter subitement, en 1979, à celui de 100 travailleurs. Certains juristes contestent, sur ce point, la légalité et la constitutionnalité de ces arrêtés royaux d'appli-

43. Rappelons qu'en 1948 le seuil était fixé à 300 travailleurs. 
cation, dans la mesure où le Roi excède son pouvoir de retarder l'entrée en vigueur de la loi.

Le seuil national, fixé à 50 par le législateur, n'a donc jamais été mis en application. La non-application de cette loi affecte fortement les modalités de transposition de la directive 2002/14/CE. En clair, le blocage institutionnel d'aujourd'hui ne se comprend qu'en regard du blocage institutionnel d'hier ou, plus exactement, en regard de l'habitude prise de ne pas mettre en œuvre la loi de 1948 qui fixe (à 50 travailleurs) le seuil pour la création d'un conseil d'entreprise. Pour le dire autrement, les intérêts qui se heurtent aujourd'hui au sein du CNT sont largement dépendants du sentier emprunté, sont largement façonnés par cette routine, ce " geste » reproduit ou actualisé tous les 4 ans depuis 60 ans.

\section{V. « i » comme idées}

Le droit ne se limite pas à un catalogue de ressources et de contraintes. Il est aussi porteur de significations multiples qui doivent être interprétées, traduites, pour produire des effets. Dans cette optique, le droit, les règles ou les institutions sont envisagés comme des orientations de sens. Le point de départ de l'analyse cognitive est sans doute l'idée toute simple selon laquelle l'objet des politiques publiques n'est pas simplement de "résoudre des problèmes " mais de construire des cadres d'interprétation du monde. Le droit (ou la politique) n'est pas seulement un espace neutre offrant aux individus la possibilité de faire ou ne pas faire, d'être ou ne pas être: il constitue aussi la proclamation publique d'un sens, c'est-à-dire d'un espace de possibilités valorisées ${ }^{44}$. Cette intuition wébérienne rejoint les théories les plus novatrices de la sociologie du droit 45 , qui considèrent le droit comme une orientation endogène de l'activité sociale (et non comme une norme exogène), une « orientation de sens « qui s'inscrit dans l'activité des personnes 46 .

\section{V.1. La directive européenne comme orientation de sens}

Penchons-nous d'abord sur l'« esprit » de la directive, les différents noyaux sémantiques qui la structurent et à partir desquels rayonnent de multiples significations. Les valeurs apparaissent dans le discours d'explicitation et de justification des droits. Ainsi, dans la directive 2002/14/CE, l'Union européenne propose des schèmes d'interprétation et de valorisation du monde social que révèlent les

44. Jean DE MunCK et Jean-François ORIANNE, «Droits sociaux et mondes possibles. L'exemple du droit européen au congé parental ", in Jean DE MUNCK et Bénédicte ZIMMERMANN (dir.), La liberté au prisme des capacités, Paris : éditions de l'EHESS, 2008, p. 263-280.

45. Claude DIDRY, « Droit, histoire et politique dans la sociologie du droit de Max Weber », in Michel CouTU et Guy Rocher (dir.), La légitimité de l'État et du droit. Autour de Max Weber, Sainte-Foix (Québec) : Les Presses de l'Université Laval, Paris: LGDJ, 2006, p. 91-114; Pierre LASCOUMES et Évelyne SERVERIN, « Le droit comme activité sociale: pour une approche wébérienne des activités juridiques", in Pierre LASCOUMES (dir.), Actualité de Max Weber pour la sociologie du droit, Paris : LGDJ, 1995, p. 155-177 ; Jacques CoMmAILLE, L'esprit sociologique des lois. Essai de sociologie politique du droit, Paris : PUF, 1994.

46. Notons que d'autres approches d'orientation wébérienne se centrent davantage sur la question de la légitimité des acteurs ou des institutions, comme par exemple les travaux de Duran (Patrice DuRAN, Penser l'action publique, Paris : LGDJ, 1999) ou encore de Le Bianic et al. (Thomas LE BIANIC et Antoine Vion [dir.], Action publique et légitimités professionnelles, Paris : LGDJ, 2008). 
" considérants" de la directive. Ces «considérants" ne sont pas encore des règles (des prescriptions), mais bien les idées, des projections, qui fondent et orientent la réglementation qui va suivre. On aurait tort de considérer qu'ils sont sans effet juridique: ils rendent intelligible le contenu des dispositions auxquelles ils renvoient, ils constituent le soubassement cognitif des normes.

En termes d'idées, de représentations, la directive 2002/14/CE vise à promouvoir le dialogue social entre les partenaires sociaux en Europe (considérant $n^{\circ} 1$ ) et à favoriser la « confiance » (par le dialogue) au sein de l'entreprise, pour assurer une gestion collective et prévisionnelle des risques (considérant $\mathrm{n}^{\circ} 7$ ) : le principal message est celui d'un travail plus démocratique dans l'entreprise au sein de l'espace européen. Notons que c'est principalement cette piste sémantique qui a motivé les acteurs de la CSC et de la FGTB à se saisir de la directive pour «forcer la barrière syndicale» ou «abaisser le seuil » pour la création d'organes paritaires au sein de l'entreprise.

Une autre idée, libellée sous la forme d'une précaution, présente un intérêt tout particulier dans le contexte belge : éviter toutes contraintes administratives, financières et juridiques qui feraient obstacle à la création et au développement de petites et moyennes entreprises (considérant $n^{\circ} 19$ ). Cette idée est au centre de la position du banc patronal mais constitue également une composante forte de la position intermédiaire du syndicat libéral, comme nous le verrons par la suite. Notons d'ores et déjà qu'à l'échelle européenne, on considère toute entreprise de moins de 250 travailleurs comme une PME; en Belgique, une PME est une entreprise de moins de 50 travailleurs.

Mais l'efficience symbolique du droit n'est pas automatique, elle dépend directement de la capacité des individus à interpréter les orientations de sens qu'il propose, à inscrire le discours juridique dans un discours culturel pour que le droit s'accorde au monde vécu des personnes ${ }^{47}$. Telle est bien la mission principale des acteurs syndicaux au sein du CNT, jouant un rôle-clé d'intermédiaire juridique: donner du sens à la directive par la mise en cohérence des significations de la directive et de la culture de la négociation collective en Belgique. Une analyse fine des arguments et justifications des acteurs permet de rendre compte de ce travail d'interprétation du droit. L'Avis $\mathrm{n}^{\circ} 1508 \mathrm{du}$ CNT, qui acte les positions divergentes des membres du Conseil sur la question de la transposition de la directive 2002/14/CE en Belgique, constitue à cet égard un document précieux pour l'analyse. Cet avis synthétise les principaux arguments qui sous-tendent les positions respectives des acteurs en présence.

\section{V.2. Les coordonnées du débat}

Comme le note Bruno Jobert ${ }^{48}$, la sélection d'un schéma d'interprétation (des orientations de sens proposées par la directive) dépend largement de la position

47. Jean DE MuncK et Jean-François ORIANNE, «Droits sociaux et mondes possibles. L'exemple du droit européen au congé parental », op. cit.

48. Bruno JOBERT, « Représentations sociales, controverses et débats dans la conduite des politiques publiques ", op. cit. 
des groupes d'acteurs dans la structure sociale, et la constitution des acteurs dans ce débat (leur identité) dépend aussi de ces schémas d'interprétation. "Toute action sociale implique [...] une opération de définition sociale de la réalité, qui est la fois constitutive de l'acteur social et prédétermine largement sa ligne de conduite ${ }^{49}$."

Selon les membres représentant la FGTB et la CSC, la demande d'avis soumise au Conseil national du travail sur la transposition de la directive touche à la question du dialogue social dans les PME. Cette interprétation "partisane» de la demande sous-tend la position commune de la FGTB et de la CSC, dont les principes de base sont les suivants; il faut: (1)définir un régime pour les PME à partir de 20 travailleurs ; (2) mettre en place un régime interne à l'entreprise avec des représentants des travailleurs; (3) prévoir la création d'un comité de prévention et de protection avec des compétences élargies aux informations économiques et financières ; (4) au moins passer par la désignation au sein des organisations syndicales ; (5) prévoir une protection normale comme celle des élus au CPPT/CE, ainsi que (6) un régime de base interprofessionnel tous secteurs confondus. En se référant à l'article 51 de la loi du 4 août 1996 sur le bien-être, les membres représentant la FGTB et la CSC sont d'avis que ces lignes de force peuvent être transposées dans la loi par un simple arrêté royal.

Notons d'emblée que les membres représentant la CGSLB souscrivent pleinement à chacun des arguments de fond (cf. supra) développés par les membres représentant la FGTB et la CSC pour instituer une présence syndicale dans les PME, et ce à tout le moins dès 20 travailleurs. Ils divergent cependant en ce qui concerne les instruments proposés. Comme l'explique ce représentant de la CGSLB : "Pour nous, il n'y a aucun doute sur la nécessité de mettre en œuvre la directive. Mais notre position diffère de la position commune de la CSC et de la FGTB. Notre approche est plus pragmatique, plus réaliste. Pour les entreprises de moins de 50 travailleurs, il suffit d'étendre la compétence de la délégation syndicale. Il n'est donc pas nécessaire d'abaisser notre seuil national pour la création d'un CE ou d'un CPPT. » La CGSLB souhaite donc trouver une solution adaptée aux spécificités des PME qui comptent entre 20 et 50 travailleurs, en faisant usage des organes existant en Belgique : le CPPT, lorsqu'il existe, ou la délégation syndicale dans le cas contraire.

Les membres représentant les organisations d'employeurs s'opposent avec force à l'interprétation large que les organisations de travailleurs souhaitent donner à la directive. Ils estiment que la Belgique satisfait parfaitement à la directive. Cependant, ils sont d'avis que le CPPT et la délégation syndicale ne sont pas compétents en matière d'information sur l'évolution récente et probable des activités de l'entreprise et sur sa situation économique : la réglementation belge devrait donc être adaptée uniquement sur ce point. Comme le confie, en entretien, un agent de l'UCM siégeant au CNT : «Les syndicats veulent utiliser la directive pour forcer la barrière syndicale au niveau des petites entreprises et abaisser les seuils pour la création d'organes paritaires (CE et CPPT). Notre position commune avec UNIZO,

49. Ibid., p. 220. 
c'est que la législation belge satisfait très largement à la directive ; il n'y a donc que quelques aménagements mineurs à apporter à notre droit social, c'est-à-dire étendre les compétences du CPPT dans les entreprises qui comptent entre 50 et 100 travailleurs. »

Une première controverse oppose deux lectures (ou visions) du système belge de concertation sociale en regard du contexte international : du côté des représentants des travailleurs, une croyance en un "retard " de la Belgique en matière d'information et de consultation des travailleurs; du côté du banc patronal, une croyance en un système suffisamment complet et complexe.

Pour justifier leur position commune, les membres représentant la FGTB et la CSC s'appuient, en effet, sur les instruments standardisés de la comparaison internationale (principalement le rapport Davignon ${ }^{50}$ ) pour souligner le "grand retard" de la Belgique sur d'autres États membres de l'Union européenne dans le domaine de la consultation des travailleurs sur des matières économiques et des matières de sécurité. Ils citent à cet égard les exemples de la Suède (où la consultation est instituée dans toutes les entreprises), l'Allemagne (dès 5 travailleurs), l'Espagne (6), les Pays-Bas (10), la France (11), le Luxembourg (15), ou encore la Grèce et la Finlande (20).

À ce comparatisme "spontané » d'objets termes à termes, les représentants du banc patronal invoquent, en quelque sorte, l'incomparabilité des systèmes nationaux, et plus particulièrement le caractère incomparablement lourd et procédurier du système de concertation sociale. Dans l'Avis $n^{\circ} 1508$, ils commencent par rappeler que la Belgique dispose déjà d'une réglementation très élaborée en matière d'information et de consultation des travailleurs. Selon eux, le législateur belge doit donc surtout veiller à ne pas imposer aux entreprises plus d'obligations que celles prévues dans la directive.

Une deuxième controverse oppose deux visions de l'impact réel (et des effets potentiels) des organes paritaires de concertation sociale en entreprise : du côté des représentants des travailleurs, on pense que les organes paritaires garantissent le respect du droit et une meilleure gestion des risques ; du côté du banc patronal, on croit que l'introduction d'organes paritaires dans les PME aurait un impact négatif sur leur développement.

En s'appuyant sur quelques données officielles 51 et quelques données d'enquêtes ${ }^{52}$, les représentants de la FGTB et de la CSC soutiennent l'idée selon laquelle la présence d'un système de concertation sociale dans une entreprise détermine dans une large mesure le respect des dispositions du droit du travail et de la législation en matière de sécurité. C'est pourquoi ils souhaitent transposer la directive 2002/14 en prévoyant la création d'un organe paritaire pour toutes les entreprises à partir de 20 travailleurs. « Nous, on croit en la concertation sociale et on sait qu'il y

50. Il s'agit du rapport «European Systems of Worker Involvement " réalisé pour la Commission européenne par un groupe d'experts conduit par le vicomte Davignon.

51. Rapports annuels de l'Administration sécurité au travail et du Fonds des accidents du travail.

52. Principalement une enquête réalisée par l'HIVA (Katholieke Universiteit Leuven, KUL), à la demande de la CSC, auprès d'un échantillon représentatif de travailleurs en PME. 
a plus de conflits et de plaintes dans les PME. [...] Pour le banc patronal, c'est la peur de l'inconnu, c'est la crainte de voir une délégation et des organes paritaires dans les PME » (représentant CSC).

Les membres UCM contestent l'interprétation des données officielles du Fonds des accidents du travail faite par les représentants des travailleurs. Selon eux, la prise en compte d'une "variable cachée" (le secteur d'activité) permet une tout autre lecture de ces données officielles : ce ne serait pas la taille de l'entreprise (et donc la présence d'organes paritaires) mais plutôt le type d'activité qui expliquerait un plus grand nombre de plaintes et d'accidents en entreprise. Selon ces représentants, la directive ne peut pas être utilisée afin d'abaisser le seuil pour au moins deux raisons différentes : (1) cet abaissement n'est pas nécessaire d'un point de vue légal : la directive permet à la Belgique de placer le seuil à 50 travailleurs (aspect technique) ; (2) il aurait un lourd impact sur la croissance des petites entreprises et sur la création d'emplois (aspect cognitif ou culturel). Comme l'expose ce représentant de l'UCM en entretien : "On n'a pas envie de voir débarquer, dans les PME, des emmerdeurs entre guillemets [les délégués syndicaux] et des travailleurs intouchables [les représentants des CE et CPPT dont l'employeur ne peut facilement se "défaire" en raison de la protection juridique liée à leur mandat]. "

Une troisième controverse porte sur l'interprétation du droit, et plus particulièrement le sens qu'il faut donner à l'article 23 de la Constitution selon lequel : «Chacun a le droit de mener une vie conforme à la dignité humaine. [...] Ces droits comprennent notamment : [...] le droit d'information, de consultation et de négociation collective.»

Les représentants de la CSC et de la FGTB soulignent que, sur la base de cet article, une procédure ad hoc de consultation et de concertation a été imposée, dans une jurisprudence "retentissante ", à des employeurs d'entreprises de moins de 50 travailleurs qui refusaient toute concertation, de manière flagrante. Ils souhaitent dès lors que le législateur, et non les tribunaux, comble cette lacune dans le droit du travail belge.

Le banc patronal s'oppose à cette référence faite à l'article 23 de la Constitution pour abaisser le seuil : "Personne ne conteste qu'il s'agit d'un droit fondamental pour tous les citoyens. Néanmoins, il est clair que le législateur n'avait pas l'intention d'affirmer que ces droits fondamentaux ne peuvent être garantis que par l'introduction d'une représentation syndicale dans chaque entreprise à partir de 20 travailleurs. D'ailleurs, si l'on pousse plus loin ce raisonnement, à savoir que ces droits individuels ne peuvent être garantis que par le biais d'une représentation syndicale, il faudrait introduire une représentation syndicale dans chaque entreprise à partir de 2 travailleurs. De plus, ce principe, qui n'est pas absolu (cf. le cas des citoyens qui ne sont pas engagés dans une relation de louage de travail), doit être concilié avec celui, plus fondamental, de la liberté d'association. »

Notons que cette argumentation par l'absurde n'a pas entièrement convaincu le Conseil d'État. En effet, dans son avis $n^{\circ} 43.912 / 1$, le Conseil d'État souligne qu'en vertu de l'article 23 de la Constitution, mais compte tenu également des principes d'égalité et de non-discrimination inscrits aux articles 10 et 11, il y aurait lieu de 
prévoir un régime minimum d'information et de consultation des travailleurs dans les entreprises comptant moins de 50 travailleurs.

Enfin, une dernière controverse - et non la moindre - porte sur l'interprétation même de la directive, en particulier des concepts d'“ entreprise » et d' "établissement». Les représentants du banc patronal soulignent le fait que la notion d' " établissement» a été introduite dans la directive européenne au cours des travaux préparatoires, à la demande de l'Allemagne. Selon eux, cette notion diffère des notions utilisées dans le droit social belge, à savoir l'entité juridique et l'unité technique d'exploitation (cf. la loi du 20/09/1948 portant sur l'organisation de l'économie, article 14). Ils estiment donc que la Belgique satisfait parfaitement à la directive en prévoyant que la directive s'applique aux " entreprises » employant au moins 50 travailleurs et situées en Belgique.

Selon Pierre-Paul Van Gehuchten et Philippe Reyniers ${ }^{53}$, il est clair que ce vif différend sur la question du seuil traduirait davantage " une divergence de fond portant sur la raison d'être même des droits à l'information et à la consultation plutôt que sur le seul problème technique qui en a permis la focalisation ». Pour sortir de l'impasse, ces juristes de l'UCL soulignent les vertus de notre bon vieux concept d' « unité technique d'exploitation » (cf. la loi de 1948) : un concept flexible et procédural qui permettrait, selon eux, de surmonter les antinomies du binôme entreprise/établissement, " une véritable procédure de découverte » en vue d'approcher le périmètre le plus adéquat au sein duquel instaurer un conseil d'entreprise ou un CPPT. "On le voit: l'unité technique d'exploitation est un concept fonctionnel qui tantôt va conduire à déconcentrer une unité juridique importante en prenant en considération, comme unité(s) technique(s) d'exploitation, chacun des sièges d'exploitation ou quelques-uns d'entre eux rassemblés à cette fin, tantôt va concentrer des entités juridiques distinctes pour remonter au niveau du commandement économique et social que représente un "groupe" 54. " Mais, semble-til, tout le monde s'accorde sur l'intérêt de ce concept. Ce qui pose problème pour la Belgique c'est que la notion d'établissement, qui signifie une unité d'exploitation (seuil de 20) dans la directive, ne signifie rien en droit belge; en revanche, depuis 1948, c'est la notion même d'entreprise qui conduit au concept d'unité technique d'exploitation (seuil de 50). Faut-il retenir l'unité de 50 ou l'unité de 20 ?

Pour Filip Dorssemont (avocat de la CSC), la Belgique n'a pas le choix : comme le législateur belge a opté pour le développement de procédures d'information et de consultation au niveau de l'unité technique d'exploitation, c'est-à-dire l'« établissement» au sens de la directive, le seuil de 20 travailleurs doit être respecté. C'est principalement sur la base de cet argument que la CSC a sollicité, le 20 avril 2006, l'intervention de la Commission européenne pour introduire un recours à la Cour européenne de justice.

Comme nous l'avons vu, la Cour européenne de justice de Luxembourg a rendu son arrêt le 29 mars 2007, et condamné la Belgique pour «manquement d'État».

53. Pierre-Paul Van GehuChten et Philippe ReYniers, Information et consultation des travailleurs. De quelques paradoxes de droit du travail à la lumière de la directive 2002/14/CE, note de travail, UCL, 2004.

54. Ibid. 
Selon la Cour européenne, la Belgique n'a pas respecté le délai de trois ans pour adapter son droit du travail : la législation belge n'accorde en effet aucun droit à des informations socio-économiques et à la concertation pour les travailleurs d'entreprises de moins de 100 salariés. Il est important de noter que l'argument juridique retenu est celui de la non-application de la loi belge de 1948 qui fixe le seuil à 50 travailleurs. En effet, depuis 1979, les arrêtés royaux d'application de cette loi, produits tous les quatre ans à l'occasion des élections sociales, déterminent le seuil à 100 travailleurs. Dès lors, même si la loi belge de 1948 est conforme à la directive, son application reste au-dessus des prescriptions minimales fixées par la Commission. Notons enfin que cet argument « décisif » retenu par la Cour européenne n'a jamais été explicitement invoqué par aucun des groupes d'acteurs en présence au sein du CNT (cf. Avis $\mathrm{n}^{\circ}$ 1508).

L'analyse des arguments montre la manière dont chaque groupe d'acteurs mobilise et interprète la directive européenne. Elle souligne le poids des représentations (des idées et des valeurs) dans la structuration des intérêts, dans le positionnement respectif des groupes en présence au sein du débat. Elle montre également l'intense travail de justification ou de rationalisation ex post de ces intérêts. Cependant, cet examen se borne à considérer les "idées " dans leur dimension substantielle, comme des contenus (intéressés et/ou valorisés) qui pèsent sur l'action. Or, l'intérêt d'une approche cognitive des politiques publiques réside également dans la dimension procédurale de l'«esprit" qui conduit directement à l'examen de processus interactifs orientés vers l'intercompréhension.

\section{VI. « i » comme interaction}

L'esprit n'est pas un produit mental localisé dans le cerveau 55 : les idées ne naissent pas dans la tête des acteurs mais se constituent dans les interactions sociales, conversations de gestes et de symboles significatifs, dans lesquelles sont impliqués les membres d'une «communauté universelle unie par des échanges de signes » 56 . Mead situe l'origine ou l'arrière-fond des valeurs, des normes et des représentations dans la communication: il pose bel et bien un primat logique (et historique) du langage sur les idées et les normes (qui déterminent, à leur tour, les intérêts). «L'univers de discours est le medium de tous les processus sociaux et, en ce sens, il est plus universel qu'eux. Mais il ne fonctionne pas tout seul : il prend appui sur des situations de coopération ${ }^{57}$."

Les arguments des acteurs syndicaux invoqués dans le débat au sein du CNT ne sont pas purement performatifs : la production discursive n'est pas entièrement

55. George H. MEAD, L'esprit, le soi et la société, Paris : PUF, 2006, p. 199. Searle a bien montré le caractère contrefactuel de cette proposition, qui n'en reste pas moins féconde sur un plan heuristique, en sociologie, pour s'extraire de toute conception monologique de l'esprit humain (John R. SEARLE, La redécouverte de l'esprit, Paris: Gallimard, 1995, p. 152). En revanche, il rejoint Mead sur la thèse du primat logique du langage par rapport aux autres institutions : «Le langage est l'institution sociale fondamentale au sens où toutes les autres présupposent le langage, alors que le langage ne présuppose pas les autres [...] » (John R. SEARLE, La construction de la réalité sociale, Paris : Gallimard, 1998, p. 84).

56. George H. MEAD, L'esprit, le soi et la société, op. cit., p. 309-310 ; p. 317 ; p. 329 ; p. 363.

57. Ibid., p. 310. 
orientée vers l'objectif de convaincre (l'adversaire, le décideur politique ou la société civile). L'échange d'idées permet également l'expression de valeurs, de croyances, de représentations du monde du travail. Mais on aurait tort de s'arrêter à cette dimension strictement substantialiste: ces «idées» ne sont pas seulement des contenus substantiels à défendre ou déployer, ce sont avant tout des formes ou schèmes d'interprétation qui permettent aux acteurs, dans un processus d'interaction (conflit/coopération), de parvenir à se comprendre ou, à tout le moins, à produire une définition commune de la situation dans laquelle ils sont impliqués. La communication ne consiste pas simplement dans l'échange d'idées abstraites, elle passe par l'adoption du point de vue de l'autre ${ }^{58}$ : communiquer, c'est avant tout chercher à se comprendre, à travers l'échange de symboles significatifs. Dans cette arène de délibération qu'est le CNT - où « il est impossible de ne pas communiquer ", diraient les chercheurs de Palo Alto -, le comportement des acteurs est largement déterminé par la communication : les prises de position respectives sont des actes de langage (relance, contrepoint, esquive, répétition, silence, etc.), des procédures, orientés vers l'intercompréhension.

Peut-on soutenir raisonnablement que les acteurs du CNT parviennent à s'entendre sur une définition commune de la situation? À première vue, le cas empirique ne semble guère favorable à la thèse de l'intercompréhension. Et pourtant, même au plus profond de leur désaccord exprimé dans l'Avis 1508, les acteurs partagent une idée susceptible de faire consensus ; elle apparaît comme le résultat contingent du processus de délibération au sein du CNT et doit impérativement être éclairée pour saisir tout à la fois le blocage institutionnel et son issue incertaine.

Par le décentrement des perspectives qu'offre la délibération, par l'adoption progressive du point de vue de l'autre, les acteurs parviennent à exprimer unanimement, ou à généraliser, une conception conflictualiste et procédurière de la négociation collective en Belgique (héritée de l'après-guerre), peu adaptée à la vie en petits groupes (le cas des PME). Et cet accord informel, cette idée commune qui a longtemps paralysé le processus de mise en œuvre, constitue aujourd'hui le soubassement cognitif d'une issue complexe et transitoire.

L'Avis $\mathrm{n}^{\circ} 1508$ actualise, en effet, une conception conflictualiste du dialogue social en Belgique : le méta-dialogue social paraît inaccessible aux acteurs en présence. Il y transparaît en outre une représentation commune des structures de concertation sociale, telles que les conseils d'entreprise, comme particulièrement lourdes et inadaptées à la réalité des PME (entreprises qui comptent moins de 50 travailleurs).

Tel est bien l'avis du banc patronal qui ne souhaite pas voir «débarquer des emmerdeurs entre guillemets " dans les PME, comme nous l'avons vu précédemment. C'est aussi la position du syndicat libéral qui propose explicitement d'adapter les structures existantes à la réalité des PME 59. Et c'est également ce qui ressort

58. Ibid., p. 363.

59. Parmi celles-ci, le comité pour la prévention et la protection au travail (CPPT) devrait, selon la CGSLB, voir ses compétences élargies aux matières économiques et sociales, dans les entreprises sans conseil d'entreprise; pour les entreprises de moins de 50 travailleurs ne disposant pas par définition d'un tel comité, la délégation syndicale constituerait une piste possible, à condition qu'un certain nombre 
de la position commune de la CSC et de la FGTB : la nécessité de constituer un "CPPT élargi » dans les entreprises qui comptent au moins 20 travailleurs. Il s'agit donc de transférer des compétences du conseil d'entreprise au sein des CPPT, en clair de traiter des matières qui concernent le collectif de travail (l'emploi, l'avenir de l'entreprise, etc.) dans un espace de dialogue initialement réservé à des questions d'ordre plus individuel qui concernent le «bien-être " du travailleur (cf. la loi de 1996).

Et personne ne fait mention explicitement de la non-mise en œuvre de la loi de 1948 dans l'Avis $n^{\circ} 1508$, comme si aucun ne souhaitait la voir appliquée à la lettre : ce silence commun, comme acte de langage, écarte donc la solution la plus simple, la plus « rationnelle», pour sortir de l'impasse (cf. l'arrêt de la Cour européenne de justice). Personne ne se satisfait de l'institution effective de conseils d'entreprise dans les entreprises de 50 travailleurs et plus. Pas même le banc patronal - partisan d'un seuil à 50 - qui préfère la présence d'un CPPT dans les entreprises qui comptent entre 50 et 100 travailleurs, où aucun conseil d'entreprise n'aurait été institué. Pour les partisans d'un seuil à 20, c'est la même option d'un CPPT «élargi » qui est mise en avant. Le conseil d'entreprise, comme institution historique du dialogue social au sein de l'entreprise, est écarté des débats par l'ensemble des acteurs, et substitué par cette institution nouvelle qu'est le CPPT.

Un examen attentif des procédures de délibération et d'argumentation permet non seulement d'interpréter le blocage institutionnel mais aussi, et surtout, de rendre compte du changement en cours, de la transformation progressive des conceptions et institutions du dialogue social en Belgique qui se joue au CNT. Le processus d'interaction (en tant qu'épreuve) a conduit les acteurs à s'entendre sur une définition de la situation. On pourrait dire, comme Niklas Luhmann ou Gunther Teubner, que le système de communication s'est auto-décrit, et que cette auto-description détermine largement l'issue du débat, par delà le positionnement respectif des acteurs en présence. Mais ce serait oublier qu'un système ne pense pas, ne ressent pas non plus, et que seuls des acteurs physiques sont capables de s'entendre. Toujours est-il que cette " auto-description " à laquelle parviennent les acteurs fait apparaître une idée nouvelle qui semble faire consensus (faire du CPPT une institution du dialogue social dans les PME), actualise une habitude commune (ne pas appliquer la loi de 1948), et appelle un intérêt commun (sortir de l'impasse pour préserver l'autonomie du dialogue social par rapport aux décideurs politiques).

\section{VII. Épilogue : une issue complexe et incertaine}

Suite à la condamnation de la Belgique par la Cour de justice de Luxembourg le 29 mars 2007, le Gouvernement a pressé les partenaires sociaux de trouver rapidement une solution. Le 23 novembre 2007, les partenaires sociaux réunis au sein du

d'éléments soient adaptés en vue de toujours garantir une mise en place et un fonctionnement " étendu et démocratique » - la CGSLB entend par là la possibilité d'être représentée dans tous les secteurs d'activité. 
« Groupe des 10 » 60 , hors de l'arène du CNT, ont conclu un accord relatif au dialogue social en Belgique. Cet accord vise explicitement deux objectifs : améliorer le dialogue social en Belgique, et éviter la poursuite de la procédure judiciaire introduite par la Commission européenne contre la Belgique pour défaut de transposition de la directive.

La solution se caractérise, d'une part, par son haut niveau de complexité juridique et, d'autre part, par son caractère transitoire. La complexité juridique ne se comprend qu'au vu de l'intensité du conflit au sein des partenaires et du monde politique sur ce dossier. Elle se mesure également au regard de l'extrême simplicité d'une solution "théorique ", rapidement écartée des débats, qui aurait consisté en une application « à la lettre » de la loi de 1948, laquelle institue des conseils d'entreprise dans toutes les entreprises à partir de 50 travailleurs. La solution apparaît également précaire, incertaine de par son caractère explicitement transitoire : elle sera soumise à évaluation fin 2010 et réexaminée par les partenaires sociaux pour l'organisation des prochaines élections sociales prévues en 2012.

L'avis 1630 du CNT portant sur le dialogue social en Belgique 61 commente l'exécution de l'accord conclu entre les partenaires sociaux au sein du Groupe des 10, qui prévoit une transposition complète de la directive par une double voie légale et conventionnelle.

Le premier volet a trait à l'information et à la consultation des travailleurs dans les entreprises employant de 50 à 99 travailleurs (sans conseil d'entreprise) et fait l'objet d'une initiative législative modifiant la loi de 1996 relative au bien-être des travailleurs : il s'agit du projet de loi complétant la transposition de la directive 2002/14/CE, déposé par le Gouvernement (Josly Piette, ministre de l'Emploi) le 26 février 2008 à la Chambre des représentants de Belgique (Doc. 52 0890/001). Ce projet de loi vise la transposition complète de la directive européenne par une extension de la compétence du CPPT (en l'absence de conseil d'entreprise), "afin d'éviter une augmentation du nombre de délégués dans les entreprises de 50 à 99 travailleurs » 62 . L'article 3 de ce projet de loi vise à transformer la section $4 \mathrm{du}$ chapitre VIII de la loi du 4 août 1996 relative au bien-être des travailleurs, en divisant les compétences des CPPT en deux sous-sections : la première reprenant les compétences générales du comité sans modification, la seconde introduisant des compétences particulières (résiduaires) pour satisfaire aux obligations de la directive européenne 63 .

60. Notons que les instances dirigeantes des organisations de travailleurs et d'employeurs se rencontrent régulièrement en dehors du CNT; elles forment alors le «Groupe des 10 » (composé de cinq représentants des travailleurs et de cinq représentants des employeurs, sous la présidence de la Fédération des entreprises de Belgique) qui est à la base de la conclusion des accords interprofessionnels, sortes de lignes directrices des politiques d'emploi et de formation.

61. Il s'agit d'un avis du CNT et du CCE produit lors d'une séance commune des conseils tenue le 27/02/2008.

62. Rapport fait au nom de la Commission des affaires sociales (doc. 52 0890/002), Bruxelles, 17 mars 2008 , p. 6.

63. Texte adopté en séance plénière et transmis au Sénat (doc. 52 0890/003), Bruxelles, 19 mars 2008 ; projet non évoqué par le Sénat lors de la session du 10/04/2008 (document législatif nº 4-659/1). 
Le deuxième volet de l'accord complète l'obligation d'informer et de consulter les travailleurs dans les entreprises de moins de 50 travailleurs et fait l'objet d'une exécution par voie conventionnelle : il s'agit de la CCT $n^{\circ} 9$ ter du 27 février 2008 modifiant la CCT $n^{\circ} 9$ du 9 mars 1972. L'article 1 complète l'article 4 de la CCT $n^{\circ} 9$ : il étend les compétences du conseil d'entreprise qui, désormais, "sera également informé et consulté préalablement par le chef d'entreprise sur les décisions susceptibles d'entraîner des modifications importantes dans l'organisation du travail ou les contrats de travail ». L'article 2 insère un nouveau chapitre V dans la CCT $n^{\circ} 9$, intitulé «Rôle de la délégation syndicale en l'absence de conseil d'entreprise dans les entreprises de moins de 50 travailleurs ". L'article 19 bis liste les informations que le chef d'entreprise est tenu de fournir à la délégation syndicale, informations issues des comptes annuels déposés auprès de la Banque nationale de Belgique (chiffre d'affaires, rémunérations, charges sociales, pensions, état des immobilisations corporelles et incorporelles et financières, bénéfice).

Lors des entretiens en 2008, les acteurs du CNT ont tous fait part de leur relative insatisfaction sur cet accord. «On a tranché sans trancher. C'est une solution complexe, à plusieurs niveaux, pour une période transitoire », explique ce représentant de la CGSLB. "C'est clair, on ne va pas sauter au plafond! Mais c'est un début. On verra en 2010 » (FGTB). Sur la question de l'application de la loi de 1948, les acteurs restent muets, visiblement mal à l'aise. Ce silence nous renvoie directement à cet accord informel et implicite qui sous-tend l'avis $n^{\circ} 1508 \mathrm{du}$ CNT, ce socle cognitif commun qui permet aujourd'hui de fonder un accord formel provisoire (l'avis $\left.\mathrm{n}^{\circ} 1630\right)$ : personne ne souhaite l'institution de conseils d'entreprise dans les entreprises de moins de 100 travailleurs. La (sur-)protection des représentants des travailleurs apparaît très certainement comme un obstacle ; le risque de voir apparaître une culture d'opposition (ou conflictualiste), peu adaptée à la vie des petits groupes, en est un autre.

L'imagination et l'apprentissage institutionnels ont progressivement permis de concevoir des modalités plus souples d'information et de consultation des travailleurs dans les PME, permettant de maintenir des capacités citoyennes au sein de l'entreprise dans une économie volatile. C'est ainsi que deux institutions du dialogue social se redéfinissent actuellement en Belgique pour répondre aux exigences minimales de la directive et promouvoir, au-delà de ces exigences, le dialogue social dans les PME : d'abord, cette institution nouvelle qu'est le CPPT, initialement chargée de la promotion et du développement du bien-être du travailleur, se voit aujourd'hui confier des missions ayant trait aux relations collectives de travail et d'emploi dans les entreprises qui comptent entre 50 et 99 travailleurs ; d'autre part, la délégation syndicale, qui par définition ne constitue pas un organe paritaire et dont le statut n'a jamais été reconnu officiellement par le législateur, se voit confier ces mêmes missions dans les entreprises de moins de 50 travailleurs.

\section{Conclusion : pour une sociologie pragmatique de l'action publique}

L'utilisation du "modèle des trois i » pour analyser la mise en œuvre d'une directive européenne en Belgique a permis de faire apparaître, et de discuter in 
concreto, deux limites de ce modèle heuristique en sciences politiques : d'une part, la faible articulation théorique entre les trois « $\mathrm{i}$ »; d'autre part, une conception substantialiste des intérêts, des institutions et des idées que ce modèle privilégie.

L'étude du cas empirique a conduit à envisager l'introduction d'un quatrième $\mathrm{i}$, transversal au modèle, comme lieu d'articulation entre les intérêts, les institutions et les idées: l'interaction, médiatisée par le langage et orientée vers l'intercompréhension. La science politique semble ignorer l'interaction, trop occupée sans doute à concilier ou dépasser l'opposition entre holisme (analyses déterministes) et individualisme (analyses stratégiques). Seule une sociologie pragmatique de l'action publique ${ }^{64}$ pourrait apporter à l'analyse des politiques publiques cette théorie de l'interaction qui lui fait cruellement défaut et qui permettrait de rendre compte de l'émergence, de l'actualisation ou de la transformation des idées, des intérêts et des institutions.

La sociologie pragmatique - directement inspirée du pragmatisme américain de Mead - accorde un statut privilégié à la dimension procédurale de la rationalité ainsi qu'à la dimension processuelle de l'action 65. Elle permet alors d'envisager : (1) les intérêts comme des procédés d'intéressement ${ }^{66}$, des procédures par lesquelles on adopte le point du point de vue d'un autrui que l'on cherche à mobiliser dans le cadre d'une négociation ; (2) les institutions comme des procédures d'apprentissage collectif par lesquelles s'actualisent et se transforment les normes ; (3) les idées comme des schèmes d'interprétation orientés vers l'intercompréhension.

Sur un plan méthodologique, cette sociologie pragmatique de l'action publique invite le chercheur à opérer un changement de perspective radical : dans cette voie, l'objet d'analyse est moins la politique publique (en tant que programme d'action gouvernementale) que les procédures concrètes par lesquelles les acteurs la font exister. Il faut donc suivre de près, non pas forcément les acteurs, mais à tout le moins les contraintes pragmatiques qu'ils éprouvent dans l'action 67 , les ressources qu'ils mobilisent, les orientations de sens qu'ils interprètent, en clair décrire les multiples intermédiations du droit ou de la politique 68 .

Ainsi revisité, le "modèle des trois $\mathrm{i}$ » cesserait de n'être qu'une signalétique pour la recherche en sciences politiques (attention, route à trois voies !) mais pourrait constituer, s'il résiste à l'épreuve des faits, une véritable théorie de l'action publique 69 .

64. Pierre Lascoumes et Patrick Le Galès, Sociologie de l'action publique, Paris : Armand Colin, 2007 ; JeanLouis GÉNARD et Fabrizio CANTELLI, Les approches pragmatiques de l'action publique, Paris : LGDJ (à paraître).

65. Mohamed NACHI, Introduction à la sociologie pragmatique. Vers un nouveau «style» sociologique?, Paris : Armand Colin, 2006.

66. François EYMARD-DuvERNAY et Emmanuelle MARCHAL, « Les règles en action : entre une organisation et ses usagers", Revue française de sociologie, XXXV (1), 1994, p. 5-36 ; Michel CALlon, "Éléments pour une sociologie de la traduction ", L'Année sociologique, 36, 1986, p. 169-208.

67. Nicolas DoDier, «Les appuis conventionnels de l'action. Éléments de pragmatique sociologique», Réseaux. Communication - Technologie - Société, 11 (62), 1993, p. 68.

68. Jean DE MunCK, "Vers un nouveau paradigme du droit», in François EYMARD-DuvernaY (dir.), L'économie des conventions. Méthodes et résultats, tome 1 : Débats, Paris : La Découverte, 2006, p. 249-264.

69. L'écriture de ce texte a grandement bénéficié des discussions que j'ai eues avec Jean De Munck, Hugues Draelants et Olgierd Kuty. J'ai également tiré parti des commentaires de mes collègues du CRIS (ULg), 


\section{口'auteur}

Jean-François Orianne est professeur à l'Institut des sciences humaines et sociales (ISHS) de l'Université de Liège et à l'Institut catholique des hautes études commerciales (ICHEC, Bruxelles), ainsi que chercheur associé au laboratoire Institutions et dynamiques historiques de l'économie (IDHE, ENS de Cachan) et à l'Observatoire social européen. Il mène diverses recherches dans le champ des politiques de l'emploi et de la formation professionnelle. Ses travaux récents, réalisés dans le cadre du réseau CAPRIGHT, portent sur les services publics de l'emploi en Europe et sur le dialogue social européen.

Il a publié récemment :

- De l'école au marché du travail, l'égalité des chances en question (dir., avec Vincent DuPRIEZ et Marie VerHOEVEN), Bruxelles : Peter Lang, 2008 ;

— «Droits sociaux et mondes possibles. L'exemple du droit européen au congé parental " (avec Jean DE MUNCK), in Jean DE MUNCK et Bénédicte ZIMMERMANN (dir.), La liberté au prisme des capacités. Amartya Sen au-delà du libéralisme, Paris : éditions de l'EHESS, coll. « Raisons pratiques, 18 », 2008.

en particulier Joël Ficet, ainsi que des intéressants débats avec les étudiants de l'ISHS qui ont suivi mon cours de sociologie de l'action publique (2008-2009). Je tiens enfin à remercier les deux lecteurs anonymes de la revue pour leurs critiques pertinentes et constructives. 